\title{
LIPSCHITZ CONSTANTS AND MODULI OF CONTINUITY FOR THE CHEBYSHEV PROJECTION
}

\author{
DOUGLAS S. BRIDGES
}

\begin{abstract}
It is shown how to construct Lipschitz constants and moduli of continuity for the Chebyshev projection of $C[0,1]$ onto the finite-dimensional subspace spanned by a Chebyshev system.
\end{abstract}

It is well known that if $X$ is a finite-dimensional linear subspace of a real normed space $E$, and if each $a \in E$ has a unique best approximant $P(a)$ in $X$, then the projection $P$ of $E$ onto $X$ is continuous (cf. [6, pp. 25-26]). We shall discuss this result in the context of Chebyshev approximation over $[0,1]$.

Although our discussion will take place entirely within the framework of Bishop's constructive mathematics [2,3], the results and estimates obtained below are also new to classical (that is, traditional) mathematics. Indeed, it is difficult to see how these estimates could have been obtained by someone working outside the constructive framework.

Let $C[0,1]$ be the space of continuous, real-valued functions on $[0,1]$ (taken with the sup norm), and let $\phi_{1}, \ldots, \phi_{N}$ be elements of $C[0,1]$. Taking $\mathbf{R}^{N}$ with the norm $\|\cdot\|_{2}: x \rightarrow\left(\sum_{i=1}^{N} x_{i}^{2}\right)^{1 / 2}$, let

$$
\phi(x)=\left(\phi_{1}(x), \ldots, \phi_{N}(x)\right) \quad(0 \leqslant x \leqslant 1)
$$

and

$$
\|\phi\|=\sup \left\{\|\phi(x)\|_{2}: 0 \leqslant x \leqslant 1\right\} .
$$

Define mappings $\beta, \kappa:\left(0, \frac{1}{N}\right] \rightarrow \mathbf{R}$ as follows: for each $\alpha \in\left(0, \frac{1}{N}\right]$,

$$
\begin{aligned}
\beta(\alpha) & =\inf \left\{\left|\phi_{1}(x)\right|: 0 \leqslant x \leqslant 1\right\} \quad \text { if } N=1, \\
= & \inf \left\{\left|\operatorname{det}\left[\phi_{j}\left(x_{i}\right)\right]\right|: 0 \leqslant x_{1}, \ldots, x_{N} \leqslant 1, \min _{1<i<j<N}\left|x_{i}-x_{j}\right| \geqslant \alpha\right\} \quad \text { if } N \geqslant 2,
\end{aligned}
$$

and

$$
\kappa(\alpha)=\max \left(1, \beta(\alpha)^{-1}\|\phi\| N^{1 / 2}(N-1) ! \prod_{i=1}^{N}\left(1+\left\|\phi_{i}\right\|\right)\right) .
$$

From now on, we shall assume that $\left\{\phi_{1}, \ldots, \phi_{N}\right\}$ is a Chebyshev system: that is, it satisfies the condition

$$
\text { for each } \alpha \in\left(0, \frac{1}{N}\right], \quad \beta(\alpha)>0 \text {. }
$$

Received by the editors May 8, 1981.

1980 Mathematics Subject Classification. Primary 41A50; Secondary 03F65.

Key words and phrases. Chebyshev system, Chebyshev projection, constructive. 
This condition is classically equivalent to, and constructively entails, the classical Haar condition: every linear combination of the functions $\phi_{1}, \ldots, \phi_{N}$ has at most $N-1$ zeroes in $[0,1]$. However, we cannot assert that $(\mathbf{H})$ and the Haar condition are constructively equivalent, as there is no known constructive proof that a continuous mapping of a compact metric space into the positive real line has positive infimum.

Let $H$ be the $N$-dimensional linear subspace of $C[0,1]$ spanned by $\left\{\phi_{1}, \ldots, \phi_{N}\right\}$. In $[5, \S 4]$ there is given a constructive proof that there exists a pointwise continuous mapping $P: C[0,1] \rightarrow H$ such that

$$
\|a-p\|>\|a-P(a)\|=\operatorname{dist}(a, H)=\inf \{\|a-h\|: h \in H\}
$$

whenever $a \in C[0,1], p \in H$ and $\|p-P(a)\|>0 . P(a)$ is called the best Chebyshev approximant of $a$ in $H$, and $P$ the Chebyshev projection of $C[0,1]$ onto $H$.

Now, although there is no proof of the Uniform Continuity Theorem that is constructive in our sense, general considerations suggest that if a constructively defined function can be proved pointwise continuous, we should be able to establish its uniform continuity on compact sets. Thus, bearing in mind that $C[0,1]$ is complete, if we are given a totally bounded subset $A$ of $C[0,1]$, we should be able to calculate a modulus of continuity for the Chebyshev projection on $A$. We do this in Theorem 2 below.

In this context, the reader should be aware that we interpret the phrase "modulus of continuity" in the manner standard in constructive mathematics: a modulus of continuity for a mapping $f$ between metric spaces $(E, d)$ and $\left(E^{\prime}, d^{\prime}\right)$ is an operation $\omega: \mathbf{R}^{+} \rightarrow \mathbf{R}^{+}$such that if $\varepsilon>0$ and if $x, y \in E$ are points of $E$ with $d(x, y) \leqslant \omega(\varepsilon)$, then $d^{\prime}(f(x), f(y)) \leqslant \varepsilon$. This interpretation is not the one common in approximation theory $[6$, p. 86].

In the following work, $\delta_{\phi}$ will be a modulus of continuity for the mapping $\phi$ introduced above.

Now recall that a totally bounded subset $A$ of $C[0,1]$ is uniformly equicontinuous: there exists a common modulus of continuity for the functions in $A$.

Lemma. Let $A$ be a totally bounded subset of $C[0,1]$. Let $\delta$ be a common modulus of continuity, and $M>0$ a common bound, for the functions in $A$. Then

$$
\omega: \varepsilon \rightarrow \min \left(\delta\left(\frac{\varepsilon}{2}\right), \delta_{\phi}\left(\varepsilon \beta\left(\frac{1}{N}\right) / 4 M N^{3 / 2}(N-1) ! \prod_{i=1}^{N}\left(1+\left\|\phi_{i}\right\|\right)\right)\right)
$$

is a common modulus of continuity for the functions $a-P(a)$, where $a \in A$.

Proof. Given $a \in A$, we have

$$
\begin{aligned}
\|P(a)\| & \leqslant\|a-P(a)\|+\|a\|=\operatorname{dist}(a, H)+\|a\| \\
& \leqslant\|a\|+\|a\| \leqslant 2 M .
\end{aligned}
$$

If $P(a)=\sum_{i=1}^{N} b_{i} \phi_{i}$, it follows from [5, Lemma 2.1] that

$$
\left(\sum_{i=1}^{N} b_{i}^{2}\right)^{1 / 2} \leqslant 2 M \beta\left(\frac{1}{N}\right)^{-1} N^{3 / 2}(N-1) ! \prod_{i=1}^{N}\left(1+\left\|\phi_{i}\right\|\right) .
$$


Let $x, y \in[0,1]$, with

$$
|x-y| \leqslant \min \left(\delta\left(\frac{\varepsilon}{2}\right), \delta_{\phi}\left(\varepsilon \beta\left(\frac{1}{N}\right) / 4 M N^{3 / 2}(N-1) ! \prod_{i=1}^{N}\left(1+\left\|\phi_{i}\right\|\right)\right)\right) .
$$

Then, using the Cauchy-Schwarz inequality in $\mathbf{R}^{N}$ and the foregoing, we have

$$
\begin{aligned}
|(a-P(a))(x)-(a-P(a))(y)| & \leqslant|a(x)-a(y)|+|P(a)(x)-P(a)(y)| \\
& \leqslant \frac{\varepsilon}{2}+\left|\sum_{i=1}^{N} b_{i}\left(\phi_{i}(x)-\phi_{i}(y)\right)\right| \\
& \leqslant \frac{\varepsilon}{2}+\left(\sum_{i=1}^{N} b_{i}^{2}\right)^{1 / 2}\|\phi(x)-\phi(y)\|_{2} \leqslant \varepsilon .
\end{aligned}
$$

As $H$ is finite-dimensional, we see from the first part of the proof of the lemma that $\{P(a): a \in A\}$ is totally bounded. Hence $S=\{a-P(a): a \in A\}$ is totally bounded and therefore uniformly equicontinuous. However, the lemma tells us much more than this, as it enables us to write down a common modulus of continuity for the functions in $S$, in terms of $\delta_{\phi}$, a bound for $A$, and a common modulus of continuity for the functions in $A$. Moreover, we can easily compute the last two quantities using the information that, for each $\varepsilon>0$, there exists a finite subset $B_{\varepsilon}$ of $A$ such that $\operatorname{dist}\left(f, B_{\varepsilon}\right)<\varepsilon$ for each $f \in A$. For we can take $M=1+$ $\max \left\{f: f \in B_{1}\right\}$; while if, for each $\varepsilon>0, \delta(\varepsilon, \cdot)$ is a common modulus of continuity for the finitely many functions in $B_{\varepsilon}$, then $\varepsilon \rightarrow \delta\left(\frac{\varepsilon}{3}, \frac{\varepsilon}{3}\right)$ is a common modulus of continuity for the functions in $A$ [3, Chapter 2, proof of 5.3].

Taken with the above lemma, a synthesis of arguments from [4 and 5] enables us l. prove

THEOREM 1. Let $A$ be a totally bounded subset of $C[0,1]$ such that $0<d=\inf \{\|a-P(a)\|: a \in A\}$. Let $\delta, M$ and $\omega$ be as in the lemma, and let $0<t \leqslant d$. Then

$$
\left\|P(a)-P\left(a^{\prime}\right)\right\| \leqslant 2 N \kappa(\alpha)\left(\sum_{i=1}^{N+1} \kappa(\alpha)^{N+i-1}-1\right)\left\|a-a^{\prime}\right\|
$$

whenever $a \in A, a^{\prime} \in C[0,1]$ and $0<\alpha \leqslant \min \left(\frac{1}{N}, \omega(t)\right)$.

Proof. Let $a \in A, a^{\prime} \in C[0,1]$ and $0<\alpha \leqslant \min \left(\frac{1}{N}, \omega(t)\right)$. Given $\varepsilon \in\left(0, \frac{1}{2} d\right)$, compute $j \in\{0,1\}$ and a strictly increasing sequence $\left(x_{1}, \ldots, x_{N+1}\right)$ of $N+1$ points of $[0,1]$ such that

$$
(-1)^{k-j}(a-P(a))\left(x_{k}\right)>\|a-P(a)\|-\varepsilon
$$

for $k=1, \ldots, N+1[4,3.4]$. For such $k$,

$$
\begin{aligned}
&(-1)^{k-j}\left(P\left(a^{\prime}\right)-P(a)\right)\left(x_{k}\right)=(-1)^{k-j}(a-P(a))\left(x_{k}\right) \\
&+(-1)^{k-j}\left(P\left(a^{\prime}\right)-a^{\prime}\right)\left(x_{k}\right)+(-1)^{k-j}\left(a^{\prime}-a\right)\left(x_{k}\right) \\
&>\|a-P(a)\|-\varepsilon-\left\|a^{\prime}-P\left(a^{\prime}\right)\right\|-\left\|a^{\prime}-a\right\| \\
&= \operatorname{dist}(a, H)-\operatorname{dist}\left(a^{\prime}, H\right)-\left\|a^{\prime}-a\right\|-\varepsilon \geqslant-2\left\|a^{\prime}-a\right\|-\varepsilon .
\end{aligned}
$$


On the other hand, for $1 \leqslant k \leqslant N$,

$$
\left|(a-P(a))\left(x_{k+1}\right)-(a-P(a))\left(x_{k}\right)\right|>2(\|a-P(a)\|-\varepsilon)>d \geqslant t,
$$

so that, by our lemma, $x_{k+1}-x_{k} \geqslant \omega(t) \geqslant \alpha$. It follows from Lemmas 4.2 and 4.3 of [5] that

$$
\left\|P(a)-P\left(a^{\prime}\right)\right\| \leqslant N \kappa(\alpha)\left(\sum_{i=1}^{N+1} \kappa(\alpha)^{N+i-1}-1\right)\left(2\left\|a-a^{\prime}\right\|+\varepsilon\right) .
$$

As $\varepsilon \in\left(0, \frac{1}{2} d\right)$ is arbitrary, the result follows.

A classical corollary of Theorem 1 is a result of Henry and Schmidt [10]: if $K$ is a compact subset of $C[0,1]$ that is disjoint from $H$, then there exists $\gamma>0$ such that $\left\|P(a)-P\left(a^{\prime}\right)\right\| \leqslant \gamma\left\|a-a^{\prime}\right\|$ whenever $a \in K$ and $a^{\prime} \in C[0,1]$. From a constructive viewpoint, it appears extremely unlikely that we could prove the latter without the additional hypothesis that $\inf \{\|a-P(a)\|: a \in K\}>0$. However, according to Theorem 1, this additional hypothesis enables us not only to assert that $\gamma$ exists, but also to describe it explicitly.

For other information on Lipschitz conditions for the Chebyshev projection, see $[1,7,8,9]$.

THEOREM 2. Let $A$ be a totally bounded subset of $C[0,1]$, and let $\delta, M$ and $\omega$ be as in the lemma. For each $\varepsilon>0$, define

$$
\begin{aligned}
& \sigma(\varepsilon)=\kappa\left(\min \left(\frac{1}{N}, \omega\left(\frac{\varepsilon}{4}\right)\right)\right) \\
& \Omega(\varepsilon)=\min \left(\frac{\varepsilon}{8}, \varepsilon / 2 N \sigma(\varepsilon)\left(\sum_{i=1}^{N+1} \sigma(\varepsilon)^{N+i-1}-1\right)\right) .
\end{aligned}
$$

Then $\Omega$ is a modulus of continuity for the Chebyshev projection on A. More precisely, if $a \in A, a^{\prime} \in C[0,1]$ and $\left\|a-a^{\prime}\right\| \leqslant \Omega(\varepsilon)$, then $\left\|P(a)-P\left(a^{\prime}\right)\right\| \leqslant \varepsilon$.

Proof. Let $a \in A, a^{\prime} \in C[0,1], \varepsilon>0$, and $\left\|a-a^{\prime}\right\| \leqslant \Omega(\varepsilon)$. Either $\frac{\varepsilon}{4}<\| a-$ $P(a) \|$ or $\|a-P(a)\|<\frac{3 \varepsilon}{8}$. In the former case, applying Theorem 1 to the totally bounded subset $\{a\}$, we obtain

$$
\left\|P(a)-P\left(a^{\prime}\right)\right\| \leqslant 2 N \sigma(\varepsilon)\left(\sum_{i=1}^{N+1} \sigma(\varepsilon)^{N+i-1}-1\right)\left\|a-a^{\prime}\right\| \leqslant \varepsilon .
$$

In the case $\|a-P(a)\|<\frac{3 \varepsilon}{8}$, we have

$$
\left\|a^{\prime}-P\left(a^{\prime}\right)\right\| \leqslant\left\|a^{\prime}-P(a)\right\| \leqslant\left\|a^{\prime}-a\right\|+\|a-P(a)\|<\frac{\varepsilon}{2} .
$$

Then

$$
\begin{aligned}
\left\|P(a)-P\left(a^{\prime}\right)\right\| & \leqslant\|a-P(a)\|+\left\|a-a^{\prime}\right\|+\left\|a^{\prime}-P\left(a^{\prime}\right)\right\| \\
& <\frac{3 \varepsilon}{8}+\frac{\varepsilon}{8}+\frac{\varepsilon}{2}=\varepsilon .
\end{aligned}
$$

Thus in both cases, $\left\|P(a)-P\left(a^{\prime}\right)\right\| \leqslant \varepsilon$.

At first sight, it may seem strange that we consider the alternative "either $\frac{\varepsilon}{4}<\|a-P(a)\|$ or $\|a-P(a)\|<\frac{3 \varepsilon}{8}$ " in the above proof. We do so in order to keep the argument fully constructive [2, Chapter 2]. 
The total boundedness of $A$ in Theorems 1 and 2 is used only to obtain $\delta$ and $M$. However, this observation does not lead to a generalization of our theorems, as every bounded, equicontinuous subset of $C[0,1]$ is totally bounded (at least from a classical point of view).

ACKNOWLEDGEMENT. The author wishes to thank the referee, whose comments on the original draft of this paper led to substantial improvements in the presentation of the final version.

\section{REFERENCES}

1. M. W. Bartelt, On Lipschitz conditions, strong unicity and a theorem of A. K. Cline, J. Approx. Theory 14 (1975), 245-250.

2. E. A. Bishop, Foundations of constructive analysis, McGraw-Hill, New York, 1967.

3. D. S. Bridges, Constructive functional analysis, Res. Notes in Math., no. 28, Pitman, London, 1979.

4. __ On the foundations of best approximation theory, J. Approx. Theory 28 (1980), 273-292.

5. A constructive development of Chebyshev approximation theory, J. Approx. Theory 30 (1980), 99-120.

6. E. W. Cheney, Introduction to approximation theory, McGraw-Hill, New York, 1966.

7. A. K. Cline, Lipschitz conditions on uniform approximation operators, J. Approx. Theory 8 (1973), $160-172$.

8. C. B. Dunham, A uniform constant of strong uniqueness on an interval, J. Approx. Theory 28 (1980), 207-211.

9. M. S. Henry and J. A. Roulier, Uniform Lipschitz constants on small intervals, J. Approx. Theory 21 (1977), 224-235.

10. M. S. Henry and D. Schmidt, Continuity theorems for the product approximation operator. Theory of Approximation with Applications (A. G. Law and B. N. Sahney, Eds.), Academic Press, New York, 1976.

Department of Mathematics, University College, Buckingham MK18 IEG, England 